doi: $10.4248 / \operatorname{IJOS} 10039$

\title{
Interaction between Schwann Cells and Osteoblasts In Vitro
}

\author{
Xiao-xiao Cai ${ }^{1}$, En Luo ${ }^{2}$, Quan Yuan ${ }^{3 *}$ \\ ${ }^{1}$ Bone Biology Research Laboratory, Children's Hospital Boston, Harvard Medical School, Boston, USA \\ ${ }^{2}$ Division of Oral Biology, Tufts University School of Dental Medicine, Boston, USA \\ ${ }^{3}$ Department of Developmental Biology, Harvard School of Dental Medicine, Boston, USA
}

\begin{abstract}
Aim Given the well-known properties of Schwann cells in promoting nerve regeneration, transplanting Schwann cells into implant sockets might be an effective method to promote sensory responses of osseointegrated implants. The aim of this study was to evaluate the interaction between Schwann cells and osteoblasts.

Methodology Schwann cells derived from the sciatic nerves of neonatal rat were co-culured with osteoblasts using Transwell inserts. The proliferation of Schwann cells in the co-culture system was evaluated using methylthiazol tetrazolium (MTT) colorimetric method. Moreover, the secretions and mRNA levels of brain-derived neurotrophic factor (BDNF) and nerve growth factor (NGF) were measured by enzyme-linked immunosorbent assay (ELISA) and quantitative real-time PCR, respectively. In order to test the effect of Schwann cells on osteoblasts, alkaline phosphatase (ALP) staining and Alizerin red staining were
\end{abstract}

performed as well.

Results Schwann cells, which were co-cultured with the osteoblasts, showed an intact proliferation during the observation period. Moreover, the gene expression and synthesis of BDNF and NGF were not impaired by the osteoblasts. Meanwhile, co-cultured osteoblasts exhibited a significant increase in the proliferation on day 3 and $6(P<$ 0.05). Co-culture of these two types of cells also led to a more intense staining of ALP and an elevated number of calcified nodules.

Conclusion These findings demonstrate that, in the in vitro indirect co-culture environment, Schwann cells can maintain their normal ability to synthesize neurotrophins, which then enhance the proliferation and differentiation of osteoblasts.

Keywords osteoblasts, Schwann cell, co-culture, proliferation, differentiation

Received Feb. 20, 2010; Revision accepted Mar. 23, 2010

\section{Introduction}

Osseointegrated dental implants have been widely used in clinics and regarded as useful and reliable treatment for the replacement of missing teeth. Studies on implant loading have shown, however, that the sensory thresholds of an implant are 10-100 times higher than those of natural teeth (Muhlbradt et al., 1989; Jacobs and van Steenberghe, 1991; Hammerle et al., 1995). Although some nerve fibers were observed in the vicinity of osseointegrated implants, and suggested to be the key elements contributing to sensory responses of external mechanical loading (Wada et al., 2001; Ysander et al., 2001), these nerve fibers were present at quite a low density, resulting in a much higher sensory threshold than that of natural teeth (Muhlbradt et al., 1989; Jacobs and van Steenberghe, 1991; Hammerle et al., 1995). Without an efficient self-protective mechanism afforded by the periodontal ligament, excessive forces may be loaded on the implant, and may result in damage to peri-implant bone (Misch et al., 2005). Therefore, improving the sensory responses of osseointegrated dental implants with a method involving use of a Schwann cell graft has been proposed (Yuan et al., 2007).

Schwann cells are the glial cells of the peripheral nerve system, and play indispensable roles during neural development and regeneration (Fros- 
tick et al., 1998; Johnson et al., 2005). In periodontal ligament, Schwann cells extend their cytoplasmic processes toward the axon terminals of Ruffini endings and wrap themselves around them. Some bioactive molecules produced by Schwann cells make an important contribution to the maturation and regeneration of periodontal Ruffini endings (Alkhamrah et al., 2003; Wakisaka and Atsumi, 2003). When a Schwann cell graft has been applied to repair peripherial nerve injuries, it has been reported to have a preferential effect on functional recovery (Hess et al., 2007; Wakao et al., 2010). Therefore, a similar performance might be anticipated when Schwann cells are transplanted into implant sockets.

To verify this hypothesis, we previously studied cytocompatibility between Schwann cells and titanium implants. Schwann cells were seeded on various titanium implant surfaces and their morphology and functions were tested. The results demonstrated that Schwann cells could proliferate well on the titanium surface, and maintain their properties in nerve regeneration (Yuan et al., 2010). Another prerequisite that must be clarified, however, is whether Schwann cells will interfere with the process of osseointegration around dental implants. As is known, osteoblasts are mainly responsible for the new bone formation around dental implants during the initial process of osseointegration. We therefore studied the effect of Schwann cells on osteoblasts, and found that the proliferation and differentiation of osteoblasts could be induced, suggesting Schwann cells transplantation should not affect the process of osseointegration (Yuan et al., 2008). We have been unable to identify, however, any report examining the effect of osteoblasts on the biological functions of Schwann cells. The purpose of the present study was to further investigate the interaction between these two kinds of cells using a novel in vitro co-culture system.

\section{Material and Methods}

\section{Primary culture of Schwann cells}

Primary Schwann cells were obtained by methods similar to those first described by Brockes et al
(Brockes et al., 1979). Briefly, the bilateral sciatic nerves of neonatal Sprague-Dawley rat pups (day 2) were dissected and washed three times with phosphate-buffered saline (PBS, pH 7.4). The epineurium of each nerve was stripped under the stereomicroscope. Nerves were cut into pieces $(<1 \mathrm{~mm})$ and enzymatically dissociated using $0.1 \%$ type I collagenase (Sigma, USA) for 30 minutes and $0.25 \%$ trypsin (Sigma, USA) for 10 minutes at $37^{\circ} \mathrm{C}$. The resulting cell suspension was centrifuged (1 $\left.000 \mathrm{r} \cdot \mathrm{min}^{-1}, 8 \mathrm{~min}\right)$, placed into $25 \mathrm{~mm}^{2}$ culture flasks (Costar Corning, USA) and incubated in Dulbecco's Modified Eagle's Medium (DMEM, Gibco, USA) supplemented with $10 \%$ fetal bovine serum (FBS, Gibco, USA) and antibiotics (penicillinstreptomycin solution). The antimitotic agent cytosine arabinoside $\left(10^{-5} \mathrm{~mol} \cdot \mathrm{L}^{-1}\right)$ was added to the culture medium 24 hours after initial plating and incubated for 2-3 days to remove fibroblasts. Finally, Schwann cells were confirmed with the S-100 protein staining technique. The number of S-100 positive and negative cells were counted at 20 randomly selected fields under a microscope to calculate the purity of the cultured Schwann cells, which was found to be more than $93 \%$.

\section{Primary culture of calvarial osteoblasts}

Osteoblasts enriched cells were enzymatically isolated from calvariae of the same rat pups where Schwann cells had been harvested by the method described preciously (Hinoi et al., 2001). Briefly, dissected calvariae were sequentially digested for 10 minutes intervals with $0.1 \%$ type I collagenase (Sigma, USA) and $0.25 \%$ trypsin (Sigma, USA) at $37^{\circ} \mathrm{C}$. Cells obtained from the second to the fourth digestions were incubated in DMEM (Gibco, USA) with antibiotics and 10\% FBS (Gibco, USA). At about $90 \%$ confluence, cells were trypsinized and subcultured. To confirm that cultures were osteoblast enriched, mineralized bone nodule formation assay and alkaline phosphatase (ALP) staining were performed (data not shown). All experiments were performed using the cells of third or fourth passage.

\section{Co-culture of Schwann cells and osteoblasts}

The co-culture system was established using 
six-well Transwell clear polyester membrane inserts with $0.4 \mu \mathrm{m}$ pore size (Costar Corning, USA). Osteoblasts and Schwann cells were seeded in the tissue culture plates and Transwell inserts, respectively, at the ratio of $1: 1$ (Group A) or $2: 1$ (Group B). After one day, Schwann cells inserts were transferred into the wells containing osteoblasts, so that two different cell types shared the culture medium but were not in physical contact. Nutrient media (DMEM with 10\% FBS and antibiotics) were changed every three days.

\section{Assessment of cell proliferation}

Cell proliferation was assessed using a methylthiazol tetrazolium (MTT, Sigma, USA) method. Briefly, $1 \mathrm{~mL}$ serum free medium and $100 \mu \mathrm{L}$ MTT solution $\left(5 \mathrm{mg} \cdot \mathrm{mL}^{-1}\right)$ were added to each sample, followed by incubation for 4 hours at $37^{\circ} \mathrm{C}$ for MTT formazan formation. The medium and MTT were then replaced by $1 \mathrm{~mL}$ dimethyl sulphoxide (DMSO) to dissolve the formazan crystals. After 30 minutes, $200 \mu \mathrm{L}$ of supernatant was transferred to microplate wells (Falcon), and the optical density was quantified against a DMSO solution blank in a microplate reader at the wavelength of $492 \mathrm{~nm}$ (HTS 7000 plus, Perkin-Elmer, USA).

\section{Enzyme-linked immunosorbent assay}

To quantify the concentration of brain-derived neurotrophic factor (BDNF) and nerve growth factor (NGF) in cell cultured supernatant, commercially available enzyme-linked immunosorbent assay (ELISA) kits were used strictly according to the manufacturer's instruction (Promega, USA). Prior to analysis, the supernatant was centrifuged to remove cell debris.

\section{RNA extraction and quantitative real-time RT-PCR}

Total RNA was extracted from each sample by the TRIZOL method strictly following the manufacturer's protocol (Invitrogen, Carlsbad, USA). The concentration and purity of freshly isolated RNA were measured at $260 \mathrm{~nm}$ using a spectrophotometer and with $A_{260 / 280}$ ratio respectively. The first-strand cDNA was synthesized from $1 \mu \mathrm{g}$ RNA with murine leukemia virusreverse transcriptase (Takara, Japan), and used for quantitative real-time PCR. Expression levels of BDNF and NGF were quantified with an ABI 7300 real-time PCR system (Applied Biosystems, USA) and SYBR green PCR reaction mix (Infinigen, USA). Primers designed in primer express 2.0 analysis software were listed in Table 1. The program used was $50^{\circ} \mathrm{C}$ for 2 minutes, $95^{\circ} \mathrm{C}$ for 10 minutes, 40 cycles of $95^{\circ} \mathrm{C}$ for 15 seconds and $60^{\circ} \mathrm{C}$ for 1 minute. Melting analysis and agarose gel electrophoresis were performed to confirm the specificity of the PCR products obtained using each primer pair. The relative expression levels of genes were analyzed using the $2^{-\Delta \Delta C t}$ method by normalizing with $\beta$-actin house-keeping gene expression, and presented in fold increase relative to the control group.

\section{ALP staining}

To test the differentiation of osteoblasts, after nine days of co-culture, osteoblasts were washed with PBS and stained with the commercial kit according to the manufacturer's instruction (Sigma, USA).

Table 1 Specific primers designed following the cDNA sequences of each gene in GenBank

\begin{tabular}{clcc}
\hline Gene & Genbank accession number & \multicolumn{1}{c}{ Primers } & Product size \\
\hline BDNF & NM_012513 & $(+)$, 5'-GGAGGCTAAGTGGAGCTGACATAC-3' & 225 bp \\
& & $(-), 5$ '-GTGCTTCCGAGCCTTCCTTTAGG-3' & \\
NGF & XM_001067130 & $(+)$, 5'-CCAAGCACTGGAACTCATACTGC-3' & 142 bp \\
& & $(-), 5$ '-CTGCTGAGCACACACACGCAG-3'; & \\
B-Actin & NM_031144 & $(+)$, 5'-CACGGCATTGTCACCAACTG -3' & 167 bp \\
& & $(-)$, 5'-TTGAAGGTCTCAAACATGATCTGG -3' & \\
\hline
\end{tabular}




\section{Alizarin red staining}

The ability of osteoblasts to form calcified bone nodules was assessed using Alizarin red staining technique. After co-culture with Schwann cells for 21 days, osteoblasts were washed, fixed in $4 \%$ formaldehyde, incubated for 10 minutes at room temperature in $1 \%$ Alizarin red solution ( $\mathrm{pH} 4.2)$, and then rinsed with PBS.

\section{Statistical analysis}

All experiments presented here were run three times with triplicate samples. Data were shown as means \pm SD, and statistically analyzed using oneway ANOVA followed by Newman-Keuls post hoc tests. Differences with a probability of occurring by chance alone of less than 0.05 were considered statistically significant.

\section{Results}

\section{Intact proliferation of Schwann cells}

To test the proliferation of Schwann cells in the co-culture system, we performed MTT assay on day 3,6, and 9. During the observation period, the OD values of each group increased steadily (Figure 1), and no significant difference was noticed among the three groups, suggesting the proliferation of Schwann cells would not be affected by the co-cultured osteoblasts.

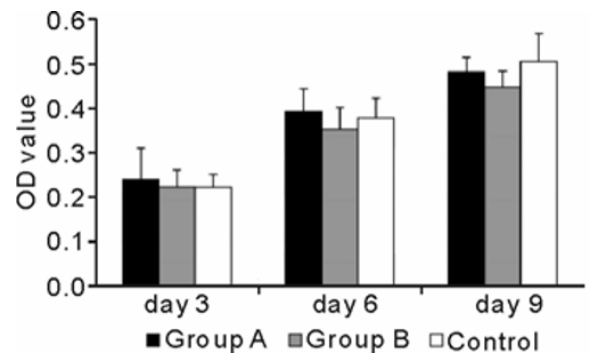

Figure 1 Proliferation of Schwann cells co-cultured with the osteoblasts

The presence of osteoblasts did not impair the proliferation of Schwann cells.

\section{Unimpaired synthesis and gene expression of BDNF and NGF in Schwann cells}

The concentrations of BDNF and NGF in the culture medium were measured on days 3, 6 and 9, and no significant difference was observed during the whole observation period (Figure 2), suggesting Schwann cells could maintain their functions of secreting neurotrophic factors even in the presence of osteoblasts. To further analyze the gene expression of these two factors, real-time quantitative PCR was performed, and it was noted that the mRNA levels of BDNF and NGF were elevated on day 6 and day 3 , respectively (Figure 2).
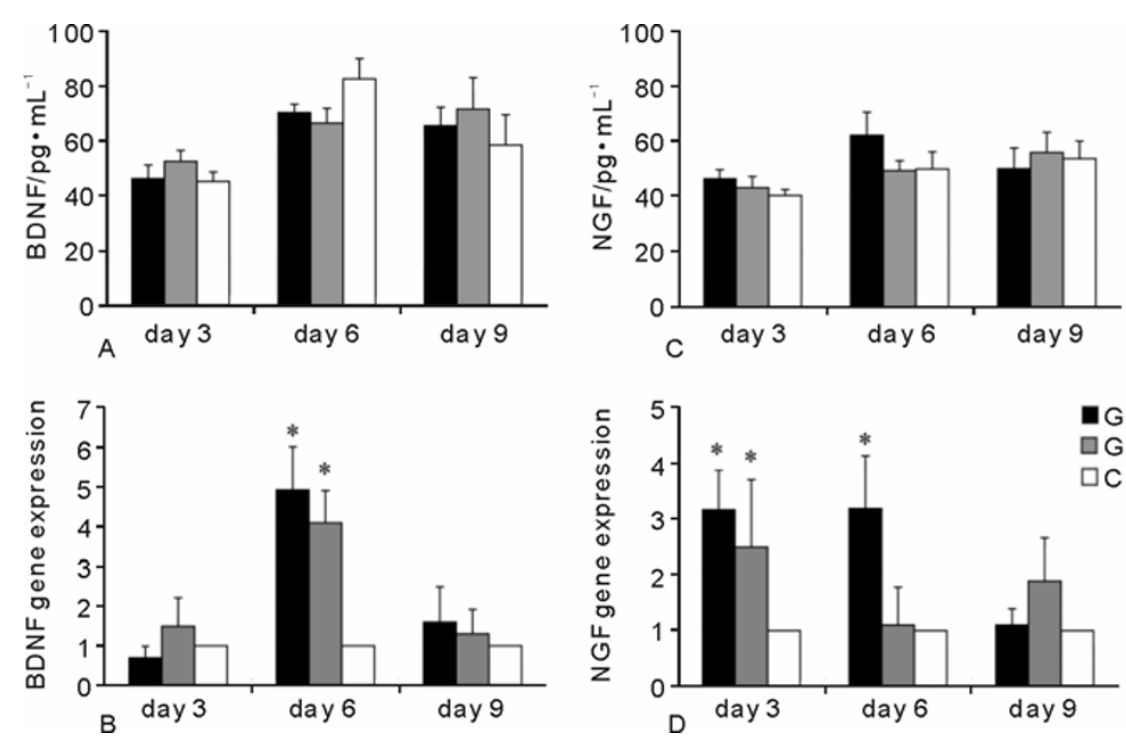

Figure 2 The effect of co-culture on synthesis and gene expression of BDNF and NGF in Schwann cells

There is no significant difference on the secretion of BDNF and NGF during the whole observation period (A and B), although the mRNA levels of BDNF and NGF were elevated on day 6 and day 3, respectively (C and D). ${ }^{*} P<0.05$. 


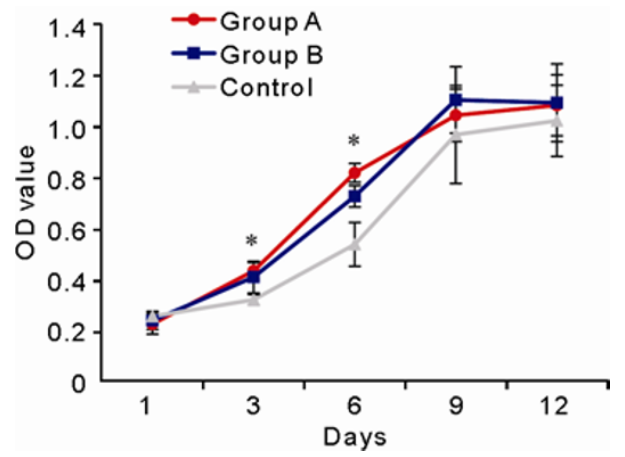

Figure 3 Proliferation of co-cultured osteoblasts measured by MTT assay

On day 3 and 6, both Group A and B had significantly higher levels of osteoblast viability than that of the control.

\section{Increased the proliferation of osteoblasts}

We further asked whether neurotrophic factors secreted by Schwann cells are biologically active. As shown in Figure 3, co-culture of Schwann cells stimulated the proliferation of rat primary osteoblasts as measured by MTT assay. On day 3 and 6, both Group A and Group B had significantly higher levels of osteoblast viability than that of the control ( $P<0.05$, see Figure 3$)$. After nine days of co-culture, cells of all three groups reached high confluence, and no significant difference was observed.
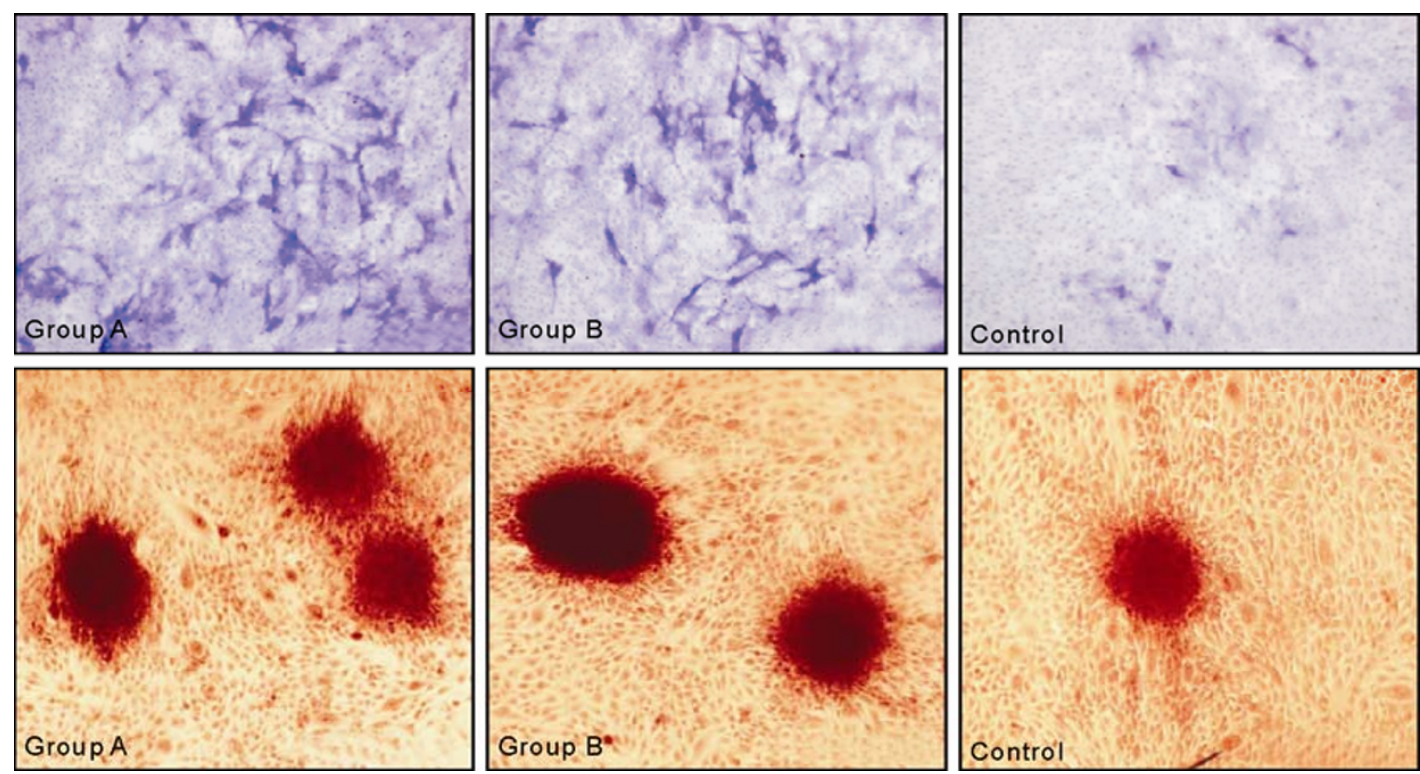

Figure 4 ALP staining (upper panel) and Alizarin red staining (lower panel) of osteoblasts

Compared with the control, co-cultured osteoblasts exhibited more intense ALP staining and many more mineralized bone nodules.

\section{Schwann cells enhance the differentiation and mineralization of osteoblasts}

To further evaluate the effect of neurotrophic factors on differentiation of osteoblats, we performed ALP staining after nine days of co-culture. As shown in Figure 4, both Group A and Group B exhibited much more intense ALP staining, suggesting Schwann cells could induce the ALP activity of osteoblasts in the indirect co-culture condition. To analyze whether Schwann cells could improve the mineralization of osteoblasts, we stained the osteoblasts with Alizarin red on day 21. As shown in Figure 4, the mineralized bone nodules were visualized by as red-purple spots. Compared with the control, both co-cultured groups had an increased number and size of calcified nodules, with more intense red staining.

\section{Discussion}

In this study, our results showed some upregulation of BDNF and NGF gene, but unimpaired secretion of BDNF and NGF peptides, which suggest that Schwann cells can maintain their functions of secreting neurotrophic factors even in the presence of osteoblasts, and these factors may 
enhance the process of osseointegration and facilitate the regeneration of nerve endings in the peri-implant region.

It is well-known that a variety of neurotrophic factors, NGF, BDNF and neurotrophin-3 (NT-3), are synthesized and released by Schwann cells (Frostick et al., 1998; Johnson et al., 2005; Bhatheja and Field, 2006). Among them, BDNF and NGF are two key neurotrophins (Frostick et al., 1998; Johnson et al., 2005; Bhatheja and Field, 2006). BDNF initiates its biological effect through binding to specific transmembrane tyrosine kinase receptor (trkB) (Bothwell, 1995), and plays an important neuroprotective role in nerve system. Alkhamrah et al. reported that the null mutant of BDNF induced a malformation and reduction of Ruffini endings in periodontal ligament (Alkhamrah et al., 2003), suggesting BDNF is required in the development and maturation of periodontal Ruffini endings. NGF is the first and best-characterized member of the neurotrophins (Wiesmann and de Vos, 2001). It plays a crucial role in regulating differentiation and survival of neurons in central and peripheral nerve systems. External NGF can enhance the regeneration of not only neural tissues, but also non-neural tissues, such as ligament, bone and periodontal membrane (Wang et al., 2006; Xu et al., 2006; Mammoto et al., 2008).

Although our data demonstrated that Schwann cells could maintain their functions of secreting neurotrophic factors in the presence of osteoblasts, whether these secreted nerothropins are biologically active are still unknown. We, therefore, evaluated their effect on the proliferation and differentiation of co-cultured osteoblasts. Consistent with our previous report (Yuan et al., 2008), osteoblasts co-cultured with Schwann cells proliferated more rapidly than osteoblasts cultured alone. Furthermore, co-cultured osteoblasts exhibited more intense staining of ALP and an elevated number of calcified nodules. These results indicated that the neurotrophic factors secreted by Schwann cells are biologically active, and could enhance the proliferation and differentiation of osteoblasts.

Some studies showed that the neurotrophins secreted by Schwann cells may bind to their receptors located on cell membrane of not only sympathetic and sensory neurons, but also various types of non-neuronal cells, including osteoblasts epithelial cells, dental pulp cells, tumor cells, and so on (Mizuno et al., 2007; Lee et al., 2008; Sigala et al., 2008; Truzzi et al., 2008). The presence of $\operatorname{trkA}, \operatorname{trkB}$ and trkC, which are the receptors of NGF, BDNF, and NT-3 respectively, has also been reported in osteoblastic cells (Nakanishi et al., 1994a; Nakanishi et al., 1994b; Asaumi et al., 2000). NGF was suggested to play an important role in the survival of osteoblastic cells (Mogi et al., 2000). In addition, Yada et al. found that NGF might promote ALP activity and collagen biosynthesis of osteoblastic MC3T3-E1 cells (Yada et al., 1994). Recently, outstanding stimulation of proliferation, ALP and COL-1 mRNA expression by NGF in human pulp cells was also reported (Mizuno et al., 2007). Our previous data also demonstrated that the presence of Schwann cells could significantly induce the expression of osteogenic marker genes, including ALP, OCN and COL-1 (Yuan et al., 2008). More importantly, in this study, we observed a much more intense staining of ALP and increased number of calcified nodules. Taken together, these findings indicate that Schwann cells enhance the differentiation of primary osteoblasts through a NGF pathway.

\section{Conclusion}

The findings of this study suggest that Schwann cells can maintain their own functions of secreting neurotrophic factors even in the presence of osteoblasts. The secreted factors are biologically active as they could improve the proliferation, differentiation and mineralization of osteoblasts. Further experiments are needed to exclude the limitation of the in vitro culture system used in this study.

\section{References}

Alkhamrah BA, Hoshino N, Kawano Y, Harada F, Hanada $\mathrm{K}$, Maeda T (2003). The periodontal Ruffini endings in brain derived neurotrophic factor (BDNF) deficient mice. Arch Histol Cytol, 66(1): 73-81.

Asaumi K, Nakanishi T, Asahara H, Inoue H, Takigawa M (2000). Expression of neurotrophins and their receptors (TRK) during fracture healing. Bone, 26(6): 625-633. 
Bhatheja K, Field J (2006). Schwann cells: origins and role in axonal maintenance and regeneration. Int J Biochem Cell Biol, 38(12): 1995-1999.

Bothwell M (1995). Functional interactions of neurotrophins and neurotrophin receptors. Annu Rev Neurosci, 18: 223-253.

Brockes JP, Fields KL, Raff MC (1979). Studies on cultured rat Schwann cells. I. Establishment of purified populations from cultures of peripheral nerve. Brain Res, 165(1): 105-118.

Frostick SP, Yin Q, Kemp GJ (1998). Schwann cells, neurotrophic factors, and peripheral nerve regeneration. Microsurgery, 18(7): 397-405.

Hammerle CH, Wagner D, Bragger U, Lussi A, Karayiannis A, Joss A, et al. (1995). Threshold of tactile sensitivity perceived with dental endosseous implants and natural teeth. Clin Oral Implants Res, 6(2): 83-90.

Hess JR, Brenner MJ, Fox IK, Nichols CM, Myckatyn TM, Hunter DA, et al. (2007). Use of cold-preserved allografts seeded with autologous Schwann cells in the treatment of a long-gap peripheral nerve injury. Plast Reconstr Surg, 119(1): 246-59.

Hinoi E, Fujimori S, Nakamura Y, Yoneda Y (2001). Group III metabotropic glutamate receptors in rat cultured calvarial osteoblasts. Biochem Biophys Res Commun, 281(2): 341-346.

Jacobs R, van Steenberghe D (1991). Comparative evaluation of the oral tactile function by means of teeth or implant-supported prostheses. Clin Oral Implants Res, 2(2): 75-80.

Johnson EO, Zoubos AB, Soucacos PN (2005). Regeneration and repair of peripheral nerves. Injury, 36 (Suppl 4): S24-S29.

Lee HW, Na YJ, Jung PK, Kim MN, Kim SM, Chung JS, et al. (2008). Nerve growth factor stimulates proliferation, adhesion and thymopoietic cytokine expression in mouse thymic epithelial cells in vitro. Regul Pept, 147(1/3): 72-81.

Mammoto T, Seerattan RA, Paulson KD, Leonard CA, Bray RC, Salo PT (2008). Nerve growth factor improves ligament healing. J Orthop Res, 26(7): 957964.

Misch CE, Suzuki JB, Misch-Dietsh FM, Bidez MW (2005). A positive correlation between occlusal trauma and peri-implant bone loss: literature support. Implant Dent, 14(2): 108-116.

Mizuno N, Shiba H, Xu WP, Inui T, Fujita T, Kajiya M, et al. (2007). Effect of neurotrophins on differentiation, calcification and proliferation in cultures of human pulp cells. Cell Biol Int, 31(12): 1462-1469.

Mogi M, Kondo A, Kinpara K, Togari A (2000). Antiapoptotic action of nerve growth factor in mouse osteoblastic cell line. Life Sci, 67(10): 1197-1206.

Muhlbradt L, Ulrich R, Mohlmann H, Schmid H (1989). Mechanoperception of natural teeth versus endosseous implants revealed by magnitude estimation. Int J Oral Maxillofac Implants, 4(2): 125-130.

Nakanishi T, Ohyama K, Aoki C, Kudo A, Hattori T, Takahashi K, et al. (1994a). Expression of trkC in a mouse osteoblastic cell line and its response to neurotrophin-3. Biochem Biophys Res Commun, 203(2): 1268-1274.

Nakanishi T, Takahashi K, Aoki C, Nishikawa K, Hattori T, Taniguchi S (1994b). Expression of nerve growth factor family neurotrophins in a mouse osteoblastic cell line. Biochem Biophys Res Commun, 198(3): 891-897.

Sigala S, Bodei S, Missale C, Zani D, Simeone C, Cunico $\mathrm{SC}$, et al. (2008). Gene expression profile of prostate cancer cell lines: effect of nerve growth factor treatment. Mol Cell Endocrinol, 284(1/2): 11-20.

Truzzi F, Marconi A, Lotti R, Dallaglio K, French LE, Hempstead BL, et al. (2008). Neurotrophins and their receptors stimulate melanoma cell proliferation and migration. J Invest Dermatol, 128(8): 2031-2040.

Wada S, Kojo T, Wang YH, Ando H, Nakanishi E, Zhang $\mathrm{M}$, et al. (2001). Effect of loading on the development of nerve fibers around oral implants in the dog mandible. Clin Oral Implants Res, 12(3): 219-224.

Wakao S, Hayashi T, Kitada M, Kohama M, Matsue D, Teramoto $\mathrm{N}$, et al. (2010). Long-term observation of auto-cell transplantation in non-human primate reveals safety and efficiency of bone marrow stromal cellderived Schwann cells in peripheral nerve regeneration. Exp Neurol, 2010 Feb 11 [Epub ahead of print].

Wakisaka S, Atsumi Y, (2003). Regeneration of periodontal Ruffini endings in adults and neonates. Microsc Res Tech, 60(5): 516-527.

Wang L, Zhou S, Liu B, Lei D, Zhao Y, Lu C, et al. (2006). Locally applied nerve growth factor enhances bone consolidation in a rabbit model of mandibular distraction osteogenesis. J Orthop Res, 24(12): 22382245.

Wiesmann C, de Vos AM (2001). Nerve growth factor: structure and function. Cell Mol Life Sci, 58(5/6): 748-759.

Xu WP, Mizuno N, Shiba H, Takeda K, Hasegawa N, Yoshimatsu S, et al. (2006). Promotion of functioning 
of human periodontal ligament cells and human endothelial cells by nerve growth factor. J Periodontol, 77(5): 800-807.

Yada M, Yamaguchi K, Tsuji T (1994). NGF stimulates differentiation of osteoblastic MC3T3-E1 cells. Biochem Biophys Res Commun, 205(2): 1187-1193.

Ysander M, Branemark R, Olmarker K, Myers RR (2001). Intramedullary osseointegration: development of a rodent model and study of histology and neuropeptide changes around titanium implants. J Rehabil Res Dev, 38(2): 183-190.

Yuan Q, Gong P, Li XY, Wei N, Tan Z, Liao DP, et al.
(2008). Enhanced proliferation and differentiation of osteoblasts induced by co-culturing with Schwann cells. Arch Med Sci, 4(3): 242-248.

Yuan Q, Gong P, Tan Z (2007). Schwann cell graft: a method to promote sensory responses of osseointegrated implants. Med Hypotheses, 69(4): 800-803.

Yuan Q, Liao D, Yang X, Li X, Wei N, Tan Z, et al. (2010). Effect of implant surface microtopography on proliferation, neurotrophin secretion, and gene expression of Schwann cells. J Biomed Mater Res A, 93(1): 381-388.

*Corresponding author: Quan Yuan

Address: 188 Longwood Avenue, Room 314, Boston, MA, 02115, USA

Tel: 16174325747 E-mail: Quan_yuan@hsdm.harvard.edu 